\title{
Consolidation Chemotherapy Prevents Relapse by Indirectly Regulating Bone Marrow Adipogenesis in Patients with Acute Myeloid Leukemia
}

\author{
Haiyan Liua Yuanmei Zhaib Wenli Zhao ${ }^{\mathrm{a}}$ Yun Wan ${ }^{\mathrm{a}}$ Wei Lu ${ }^{\mathrm{a}}$ Shaoxin Yanga \\ Yehua Yu ${ }^{d}$ Yanyu Wei ${ }^{a}$ Zhiqiang $\mathrm{Li}^{\mathrm{e}}$ Jun Shia \\ aDepartment of Hematology, Shanghai Jiao Tong University Affiliated Sixth People's Hospital, Shanghai, \\ ${ }^{b}$ Department of Hematology, Shanghai Jiao Tong University School of Medicine Affiliated Tongren \\ Hospital, Shanghai, 'Department of Hematology, Shanghai Jiao Tong University Affiliated Sixth People's \\ Hospital South Campus, Shanghai, dDepartment of Hematology, Shanghai Jiao Tong University School \\ of Medicine Affiliated Ninth People's Hospital, Shanghai, eDepartment of Blood Transfusion, Shanghai \\ Jiao Tong University Affiliated Sixth People's Hospital, Shanghai, China
}

\section{Key Words}

Acute myeloid leukaemia • Bone marrow adipocytes • Relapse • Chemotherapy • GDF15

\begin{abstract}
Background/Aims: Chemotherapy is still the main strategy used to prevent the relapse of acute myeloid leukaemia (AML). As the most abundant stromal component in bone marrow (BM), marrow adipocytes have been previously shown to promote leukaemogenesis. The present study was designed to further validate whether marrow adipocytes exert synergistic effects on strengthening chemotherapeutic efficacy and evaluate the underlying mechanism. Methods: A retrospective study of BM biopsies from 80 patients with $A M L$ in remission and 71 control subjects was applied to quantitatively analyse the marrow adipocyte volume. Toxicity tests were used to assess the effect of chemotherapy drugs on BM cells. The possible mechanisms by which chemotherapy regulated the reduced marrow adipocyte content were investigated using antibody neutralization experiments, with an emphasis on growth differentiation factor 15 (GDF15). Results: In our study, the marrow adipocyte content was obviously reduced in the AML- complete remission $(C R)$ group compared with the control group $(P<0.001)$. Moreover, patients with a reduced adipocyte content exhibited longer relapse-free survival (RFS) $(\mathrm{P}<0.001)$. We also confirmed that GDF15 was overexpressed in mononuclear cells (MNCs) after treatment with chemotherapy drugs and partially blocked mesenchymal stem cells (MSCs) adipogenesis. Intriguingly, this inhibitory effect on adipogenesis was rescued by treatment with a neutralizing anti-GDF15 antibody. Conclusion: Chemotherapy indirectly inhibited adipogenesis by promoting GDF15 secretion from BM MNCs, subsequently strengthening the efficacy of consolidation chemotherapy in patients with AML during CR.

H. Liu, Y. Zhai and W. Zhao contributed equally to this work.


Liu et al.: Chemotherapy Prevents AML Relapse by Indirectly Regulating Adipogenesis

\section{Introduction}

Acute myeloid leukaemia (AML) is a type of myeloid malignant hyperplasic disease characterized by clonal expansion of blasts in the bone marrow (BM). Induction chemotherapy enables the majority of patients with AML to achieve complete remission (CR). However, relapse after CR remains the main cause of death in patients with leukaemia $[1,2]$. According to recent studies, the relapse of some fatal haematological malignancies is closely related to the BM microenvironment $[3,4]$. As an important component of the BM microenvironment, the number of marrow adipocytes is dramatically decreased in patients with primary acute lymphoblastic leukaemia(ALL) and support ALL cell proliferation and chemo-resistance in the limited BM space [5]. Moreover, obesity is associated with a $50 \%$ increase in the recurrence rate in children with ALL [6]. These studies suggest a supportive role for adipose tissue in multiple steps of carcinogenesis. However, the complex impact of adipose tissue on AML recurrence remains unclear.

In healthy adults, adipose tissue in the iliac crest occupies $20-40 \%$ of the marrow cavity and this proportion reaches up to $80 \%-90 \%$ in the long bones [7]. Generally, the marrow adipocyte volume is measured by BM biopsy, which is not a routine inspection programme used for patients with leukaemia. A clinical diagnosis usually considers the presence of less than $5 \%$ of blasts in an aspirate sample with marrow spicules and normal haematopoietic recovery as the criterion for CR in patients with AML [8]. Researchers have not determined whether the marrow adipocyte volume is restored to the normal level, and little attention has been paid to BM adipocytes in the remission stage.

BM leukaemia cells almost disappear from patients with AML during CR, and the regulatory factors may also differ from those involved in normal $\mathrm{BM}$ and tumorigenesis. Moreover, patients with AML who have achieved CR after receiving induction therapies still require up to 6 cycles of the consolidation therapy with cytarabine, an alternative anthracycline that is mainly used to treat leukaemia. Normal haematopoietic cells and stromal cells are also exposed to chemotherapeutic drugs for long period to eliminate minimal residual disease (MRD). Regular doses of chemotherapeutic drugs mainly kill haematopoietic stem cells but are not sufficiently potent to affect BM stromal cells [9]. During chemotherapy, a large number of cytokines are secreted by haematopoietic cells [10]. Multiple factors have the potential to influence the adipogenic process, such as leptin, Interleukin 1 beta (IL-1 $\beta$ ), tumor necrosis factor alpha (TNF- $\alpha$ ), and transforming growth factor- $\beta$ (TGF- $\beta$ ) [11-13]. As a vital member of the TGF- $\beta$ family, GDF15 is predominantly expressed in the placenta and various other tissues, and it may play a dual role in tumorigenesis during different stages of the disease [14-19]. Furthermore, as shown in the study by Mika et al., GDF15 expression is induced by drugs and markedly inhibits the adipocyte differentiation of mouse 3T3-L1 cells [20]. Thus, we hypothesize that marrow adipogenesis in remission period may be regulated by chemotherapy, which may play an important role in the relapse of leukaemia, and targeted interference in BM adipocytes may provide a new therapeutic strategy for AML.

In the current study, we focus on the abnormalities in the marrow adipocyte content in patients with AML during CR and discuss the possible mechanisms by which chemotherapy drugs direct or indirect regulate adipocytes, as well as their effects on the prognosis of patients with AML. Our results may provide a reasonable explanation for why consolidation chemotherapy is still needed after remission.

\section{Materials and Methods}

\section{Samples}

BM trephine biopsy specimens from 80 patients with AML during CR and 71 controls with newly diagnosed lymphoma without BM infiltration were retrospectively analysed in this study. Patients were excluded from the study if they had been treated for less than 6 months prior to voluntarily withdrawal from therapy or died from a disease other than leukaemia. We also excluded patients who had any prior history 


\section{Cellular Physiology Cell Physiol Biochem 2018;45:2389-2400 \begin{tabular}{ll|l} 
and Biochemistry & $\begin{array}{l}\text { DOI: 10.1159/000488225 } \\
\text { Published onlIne: March 16, } 2018\end{array}$ & $\begin{array}{l}\text { (c) } 2018 \text { The Author(s). Published by S. Karger AG, Basel } \\
\text { www.karger.com/cpb }\end{array}$ \\
\hline
\end{tabular}}

Liu et al.: Chemotherapy Prevents AML Relapse by Indirectly Regulating Adipogenesis

of bone or BM disease or who had previously received treatment for malignancy. Patients were diagnosed according to the French-American-British (FAB) classification. CR and relapse were diagnosed according to the criteria reported by Cheson et al. [21]. For induction therapies, patients with AML were treated with one anthracycline agent (daunorubicin, idarubicin, epirubicin or mitoxantrone) for 3 days and cytarabine (Ara-C) for 7 days. For consolidation therapies, patients with AML received up to 6 cycles of induction therapies including one alternative anthracycline. A BM biopsy was performed at the first post-induction check-up on day 21-28 post-induction. Patients in CR were divided into the relapse and non-relapse groups according to whether patients relapsed during the follow-up period. This study was approved by the institutional Ethics Committees of Shanghai Jiaotong University affiliated hospitals and was conducted in accordance with the ethical guidelines of the Declaration of Helsinki.

\section{Adipocyte measurement}

BM trephine biopsies were obtained from the posterior iliac crest, and paraffin or plastic-embedded BM tissue slices were generated after fixation and decalcification using conventional methods. BM sections were observed under an optical microscope imaging system (Olympus BX41, Tokyo, Japan). Ten fields were randomly selected at 100x magnification and analysed at $400 \mathrm{x}$ magnification. Fields were selected from region located as close to the whole biopsy specimen as possible. The grid point overlaying the cytoplasm of fat cells or the border was considered as a fat cell-positive point. All biopsies were examined in a blinded manner without knowledge of the group to avoid any bias in the final analysis. Clinical outcomes were assessed by determining relapse-free survival (RFS) and overall survival (OS). The characteristics of the study population are presented in Table 2 .

\section{Cell culture and MSCs adipogenic differentiation assay}

Human BM mononuclear cells (MNCs) and mesenchymal stem cells (MSCs) were isolated from controls by Ficoll-Hypaque (Axis-Shield Diagnostics, Dundee, Scotland, UK) density-gradient centrifugation after informed consent was obtained.

MSCs were cultured with human adipogenic differentiation basal medium (Cyagen Biosciences Inc., CA, USA) to trigger adipogenesis as previously described [22]. The process was performed for approximately 21 days. Then, adipocytes were stained with Oil Red O (ORO) to visualize lipids, and the adipogenic capacity was analysed by measuring the optical density (OD) values at A492 nm. Additionally, levels of adipocytespecific genes $(P P A R \gamma, F A B P 4$, and $C / E B P \alpha)$ were quantitatively assessed.

\section{Drug toxicity test and EDU assay}

As a type of anti-metabolite drug, cytarabine (Ara-C, Sigma, St. Louis, Missouri, USA) is widely used in the clinical as treatment for leukaemia. A CCK8 kit was used to evaluate cell viability. MSCs were treated with or without Ara-C during the process of concomitant lipid differentiation, and then the differentiated adipocytes were chemically stained with ORO.

Approximately $1 \times 10^{5} \mathrm{MSC}$ were seeded in a 24-well plate, incubated for $48 \mathrm{~h}$, labelled with 5-ethynyl2 '-deoxyuridine (EDU) for $2 \mathrm{~h}$ and stained with Hoechst to compare the proliferation of MSCs treated with or without Ara-C. The EDU (red) and Hoechst (blue) fluorescence was detected with a fluorescence microscope (Olympus, Tokyo, Japan).

Table 1. Sequences of the primers used to detect genes expression by qPCR

\begin{tabular}{|c|c|}
\hline Primer & Primer Sequence \\
\hline $\mathrm{C} / \mathrm{EBP} \alpha$ & Forward:5'-GAACAGCAACGAGTACCGGGTA-3' Reverse:5'- GCCATGGCCTTGACCAAGGAG-3' \\
\hline PPAR $\gamma$ & $\begin{array}{c}\text { Forward:5'- GGGATCAGCTCCGTGGATCT -3' } \\
\text { Reverse:5'- TGCACTTTGGTACTCTTGAAGTT -3' }\end{array}$ \\
\hline FABP4 & Forward:5'-GCTTTGCCACCAGGAAAGTG-3' Reverse:5'-ATGGACGCATTCCACCACCA-3' \\
\hline GDF15 & $\begin{array}{l}\text { Forward:5'-GACCCTCAGAGTTGCACTCC-3' } \\
\text { Reverse: 5'-GCCTGGTTAGCAGGTCCTC-3' }\end{array}$ \\
\hline CTGF & Forward:5'-GGAGTCCACTGGCGTCTTC-3' Reverse:5'-GCTGATGATCTTGAGGCTGTTG-3' \\
\hline TGF $\beta$ & $\begin{array}{l}\text { Forward:5'-ACTTGCACCACCTTGGACTTC-3' } \\
\text { Reverse:5'- GGTCATCACCGTTGGCTCA-3' }\end{array}$ \\
\hline $\begin{array}{l}\text { LRP5 } \\
\text { GAPDH }\end{array}$ & $\begin{array}{c}\begin{array}{c}\text { Forward:5'-GGAGTCCACTGGCGTCTTC-3' } \\
\text { Forward:5'-CGGAGTCAACGGATTTGGTCGTAT-3' }\end{array} \\
\text { Feverse:5'-GCTGATGATCTTGAGGCTGTTG-3' } \\
\text { Reverse-AGCCTTCTCCATGGTGGTGGAAGAC-3' }\end{array}$ \\
\hline
\end{tabular}




\section{Cellular Physiology Cell Physiol Biochem 2018;45:2389-2400

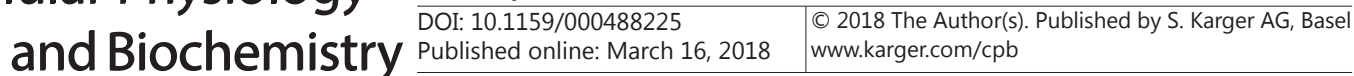

Liu et al.: Chemotherapy Prevents AML Relapse by Indirectly Regulating Adipogenesis

Flow cytometry analysis (FACS)

MNCs were separated from the BM aspirates obtained from the controls and treated with $2 \mu \mathrm{M}$ Ara-C. Then, cells were labelled with a PE-Cy7-CD45 (Biolegend, San Diego, CA, USA) antibody. CD45+ cells were sorted and double stained with Annexin V-FITC and propidium iodide (PI) (Invitrogen Life Technologies, Carlsbad, California, USA) to analyse apoptosis, according to the manufacturer's instructions.

\section{Microarray analysis}

MNCs from the controls that had been treated with or without Ara-C were used for this test, according to the manufacturer's instructions. Briefly, total RNA was isolated using an RNeasy micro kit (Qiagen), and one cycle of cDNA synthesis and linear amplification was performed. Then, in vitro transcription and labelling with biotinylated nucleotides were performed according to the Affymetrix "one-cycle" protocol. Biotinylated cRNAs were hybridized to the HGU133 plus 2.0 GeneChip arrays (Affymetrix). Chips were subsequently scanned using the GeneChip Scanner 3000(Affymetrix). Red and green signals indicate high and low levels of expression of each gene, respectively.

\section{Quantitative real-time polymerase chain reaction ( $q$ RT-PCR)}

RNA samples were extracted using TRIzol reagent (Invitrogen, Paisley, UK), and the RNA was converted into cDNA using the Primer Script TM RT Reagent Kit (TaKaRa Bio Inc., Otsu, Shiga, Japan). The PCR reactions were performed using the ABI 7500 Real-Time PCR System (Biosystems, Foster City, CA, USA) and the SYBR

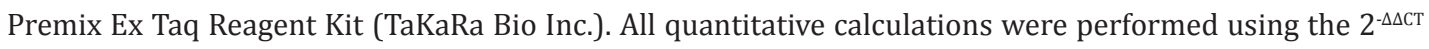
method. Primer sequences are provided in Table 1.

\section{Western blotting and ELISA}

MNCs were harvested and lysed. Protein concentrations were determined using the BCA protein assay kit (Beyotime, Haimen, Jiangsu, China). Proteins bands were visualized with an enhanced chemiluminescence reagent using a western blotting detection system (Pierce, Waltham, MA, USA). The rabbit anti-GDF15 antibody was obtained from Abcam (Cambridge, MA, USA). The horseradish peroxidase-conjugated secondary antibody was purchased from Proteintech Group, Inc. (Chicago, IL, USA). BM aspirates were collected from patients with AML-CR and the control group. The ELISA for GDF15 was performed according to the manufacturer's instructions (R\&D Systems, Minneapolis, MN, USA).

\section{Neutralizing antibody assay}

During the adipogenic process, cells were treated with human recombinant GDF15 (100ng/mL, Peprotech, Rocky Hill, NJ, USA) or a neutralizing anti-GDF15 antibody ( $4 \mu \mathrm{g} / \mathrm{mL}, \mathrm{R} \& \mathrm{D}$, Minneapolis, MN, USA) in induction medium at the indicated doses for the indicated time periods.

\section{Immunohistochemistry (IHC) assay}

BM tissue samples were used for immunohistochemistry. Sections were incubated with the rabbit antiGDF15 monoclonal antibody (Abcam, Cambridge, MA, USA) according to the manufacturer's instructions. Images of the slides stained for the marker were scanned at $400 \times$ and $1000 \times$ magnifications using an optical microscope (Olympus, Tokyo, Japan).

\section{Statistical analysis}

The chi-square test or Fisher's exact test was utilized to examine differences in categorical variables and clinicopathological characteristics between the AML-CR group and the control group. Student's test was used to compare means between two groups from in vitro experiments. Spearman's correlation test was used to analyse the correlation between the adipocyte volume and BM blast percentage. Hazard ratios (HR) and 95\% confidence intervals (CI) for different groups were estimated using a Cox regression analysis. Differences in RFS and OS between different groups were assayed with the log-rank test, and survival curves were plotted using the Kaplan-Meier method. A receiver operating characteristic curve (ROC) analysis was performed and the corresponding area under the curve (AUC) was calculated to determine the value of the adipocyte content in predicting recurrence. The data were calculated as mean \pm standard deviation (SD), and $p$ values $\leq 0.05$ were considered significant. All statistical analyses were conducted by using the SPSS 18.0 software program (SPSS Inc., Chicago, IL, USA). 


\section{Cellular Physiology Cell Physiol Biochem 2018;45:2389-2400 \\ and Biochemistry Published onlıne: March 16, $2018 \quad$\begin{tabular}{l|l} 
DOI: 10.1159/000488225 2018 The Author(s). Published by S. Karger AG, Basel \\
www.karger.com/cpb
\end{tabular}}

Liu et al.: Chemotherapy Prevents AML Relapse by Indirectly Regulating Adipogenesis

\section{Results}

\section{Patient characteristics}

The ages of the patients in the relapse and no-relapse groups were $52.21 \pm 17.50$ years and $49.88 \pm 16.41$ years, respectively. The male/female ratios in the two groups were $23 / 19$ and $20 / 18$, respectively. Among the 80 patients with AML-CR, 42 patients were allocated to the remission group because they achieved CR after induction chemotherapy and 38 patients belonged to the refractory group according to the criterion in the report from Schmid $\mathrm{C}$ and colleagues [23]. No significant differences in the basic characteristics of patients, such as age, sex or FAB subtype, were observed between the two groups (Table 2).

\section{A reduced marrow adipocyte volume during CR predicted a good prognosis of patients} with $A M L$

Staining in BM biopsies from patients with AML-CR was compared with controls to explore the marrow adipocytes volume in patients with AML during the remission phase. Adipocytes were confirmed by IHC staining for fatty-acid binding protein 4 (FABP4) and showed no obvious differences between the two groups. As shown in Fig. 1A, adipocytes were mostly round and unequal in size in the AML-CR and control groups (Fig. 1A). However, a significantly lower marrow adipocyte volume was observed in the BM sections from patients with AML-CR than from the controls (average $21.10 \%$ and $27.43 \%$; $p<0.01$ Fig. 1B), but the volume was higher than in leukaemia patients at diagnosis (data was not shown). The adipocyte volume of the same patients was compared between consolidation chemotherapy and maintainable remission to avoid potential effects of time on the functional recovery of the MSCs adipogenic differentiation capacity after remission, A difference in the adipocyte content was not observed in patients with AML-CR who underwent long-term observations during the consolidation and the maintainable remission phases $(n=8$, Fig. $1 \mathrm{C})$, indicating that the marrow adipocytes abnormality persisted during the CR period.

The prognostic impact of the adipocyte volume in 80 patients with AML-CR was explored using the Cox regression analysis to determine whether the adipocyte content was a risk factor for the survival of patients with AML-CR. Only the adipocyte volume was associated with a high risk of relapse in patients with AML-CR (Table 3). The ROC analysis of the recurrence prediction suggested that the threshold value of the adipocyte volume was $21.5 \%$ to achieve the maximum sum of both sensitivity and specificity, and this volume was the strongest predictor of a leukaemic relapse. Additionally, the probabilities of RFS were compared between patients with different adipocyte content using the Kaplan-Meier survival analysis. The results showed that patients with lower adipocyte content (adipocytes volume $\leq 21.5 \%$ ) had longer RFS and OS than other patients (Fig. 1D and 1E). Furthermore, the average adipocyte content of patients who experienced a relapse was $24.5 \%$, which was higher than the content in the patients who achieved sustained remission (Fig.1F, $\quad{ }^{* *} \mathrm{p}<0.01$ ). These results further confirmed that a decreased adipocyte volume during remission was closely related to a good prognosis of leukaemia.

Chemotherapy does not directly inhibit adipogenic differentiation Marrow adipocytes are derived from MSCs. We examined the viability of MSCs treated with Ara-C using a CCK8 assay in vitro to eliminate the possibility of toxic effects of chemotherapy drugs on MSCs and

Table 2. Characteristics of patients with AML-CR. FAB: French-American-British classification

\begin{tabular}{lccc}
\hline Characteristic & $\begin{array}{c}\text { Non-relapse } \\
(\mathrm{n}=42)\end{array}$ & $\begin{array}{c}\text { Relapse } \\
(\mathrm{n}=38)\end{array}$ & P value \\
\hline Age(years) & & & 0.542 \\
Mean \pm SD & $49.88 \pm 16.41$ & $52.21 \pm 17.50$ & \\
Range & $21-80$ & $18-77$ & \\
Sex(male/female) & $23 / 19$ & $20 / 18$ & 0.849 \\
FAB subtype M0 & 2 & 1 & 0.732 \\
M1 & 3 & 2 & \\
M2 & 10 & 8 & \\
M3 & 12 & 7 & \\
M4 & 10 & 9 & \\
M5 & 3 & 7 & \\
M6 & 2 & 3 & \\
M7 & 0 & 1 & \\
\hline
\end{tabular}


Fig. 1. The total volume of BM adipocytes is reduced in in patients with AML during CR. (A) Images of H\&E staining and immunohistochemistry with an anti-fattyacid binding protein 4 (FABP4) antibody in $\mathrm{BM}$ sections from the controls (Ctrl) and patients with AML-CR (CR), at 400x magnification. (B) Quantitative analysis of the adipocyte volume in the controls $(n=71)$ and patients with AML in remission $(\mathrm{n}=80) .{ }^{* * *} \mathrm{p}<0.001$. (C) Comparison of the adipocyte volume between patients with AML-CR during consolidation chemotherapy and maintainable remission $(n=8)$. (D) Kaplan-Meier curves showing the RFS of patients with AML in remission presenting with reduced adipocyte

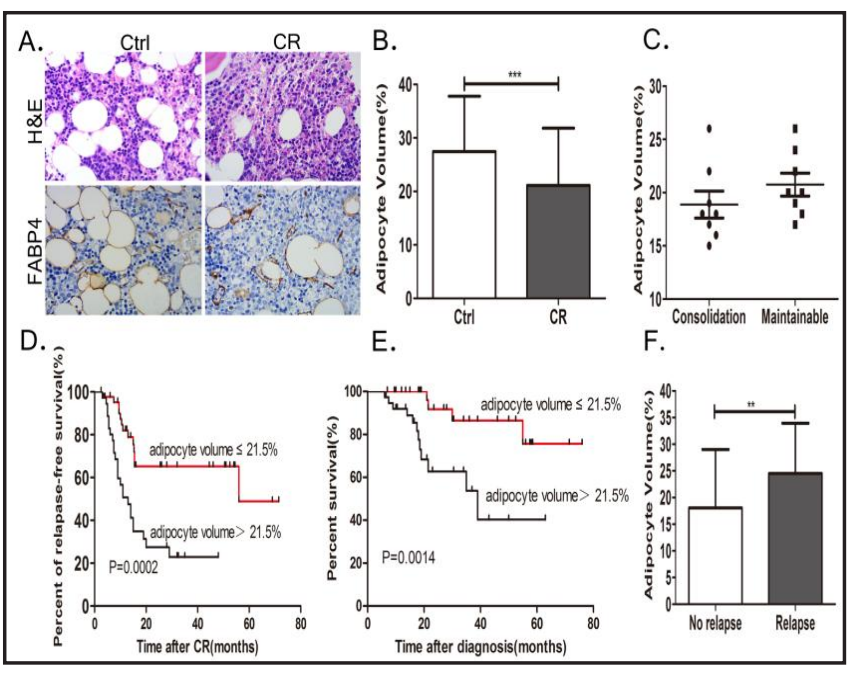
volume (adipocyte volume $\leq 21.5 \%$ ) or an increased adipocyte volume (adipocyte volume>21.5\%). (E) Kaplan-Meier curves showing the OS of patients with AML in remission presenting with a reduced adipocyte volume (adipocyte volume $\leq 21.5 \%$ ) or an increased adipocyte volume (adipocyte volume $>21.5 \%$ ). (F) Quantitative analysis of the adipocyte volume in the non-relapse $(n=42)$ and relapse patients who were in remission $(\mathrm{n}=38) .{ }^{* * *} \mathrm{p}<0.001$.

MSCs-derived adipocytes. The conventional dose of the chemotherapeutic drugs was not sufficient to kill the MSCs, and 2 $\mu \mathrm{M}$ Ara-C increased the inhibitory effect on MNC growth to nearly 50\% (Fig. 2A). Additionally, according to the results of the EDU assay, MSCs were primarily activated, and no obvious difference was observed in MSCs treated with or without Ara-C (Fig. 2B). Moreover, based on the results of BODIPY staining and the apoptosis analysis, the viability of the mature adipocytes was not affected by chemotherapeutic drugs (Fig. 2C and 2D). Then, we tested whether the changes in the adipocyte volume in patients with AML-CR were induced by the toxic effects of Ara-C during the adipogenic differentiation process. The adipogenic differentiation potential of MSCs was not impaired by the treatment with Ara-C (Fig. 2D and 2F). Thus, the reduced marrow adipocyte content in patients with AML-CR was not caused by the effects of chemotherapy on the MSCs or MSCs-derived adipocytes, or on the adipogenic capability of MSCs. Therefore, we considered that other factors might impact the adipocyte content.

\section{Conditioned medium from MNCs treated with cytarabine inhibits adipogenesis}

Continuous chemotherapy was the major difference between patients with AML-CR and the controls. Thus, we explored whether other pathways mediated the effects of consolidation chemotherapy on the adipocyte content. Because haematopoietic cells account for the majority of cells in BM during CR, we hypothesized that this effect may be related to haematopoietic cells treated with chemotherapy. The proportion of cells positive for the leukocyte common antigen CD45 was counted to confirm the relevance of BM cells and adipogenesis. Approximately $90.97 \%$ of the BM cells were $\mathrm{CD}^{4} 5^{+}$cells (Fig. 3A), indicating that MNCs might participate in the adipogenic process. However, MNCs cannot be cultured long-term in vitro, and thus, 
Fig. 2. Chemotherapy does not directly inhibit adipogenic differentiation. (A) The rate at which cytarabine (Ara-C) inhibits MSCs and MNCs proliferation. (B) EdU (red) and Hoechst (blue) staining were used to evaluate the proliferation of BM MSCs treated with or without Ara-C $(2 \mu \mathrm{M})$. Images were obtained at 200× magnification. (C and D) BODIPY staining and Apoptosis analysis of mature adipocytes treated with or without Ara-C $(2 \mu \mathrm{M})$. Images were obtained at $200 \times$ magnification. (E) The lipid differentiation process was successfully determined by ORO staining. OD values for the control and cytarabine-treated groups were analysed. Images were obtained at $200 \times$ magnification. (F) qPCR analysis of the expression of adipocyte-specific genes ( PPAR $\gamma, \mathrm{FABP} 4$ and $\mathrm{C} / \mathrm{EBP} \alpha$ ) in mature adipocytes treated with or without Ara-C $(2 \mu \mathrm{M})$. All data are presented as the mean \pm SD of triplicates samples from each independent exprement. The experiments were repeated at least three times with the same results.

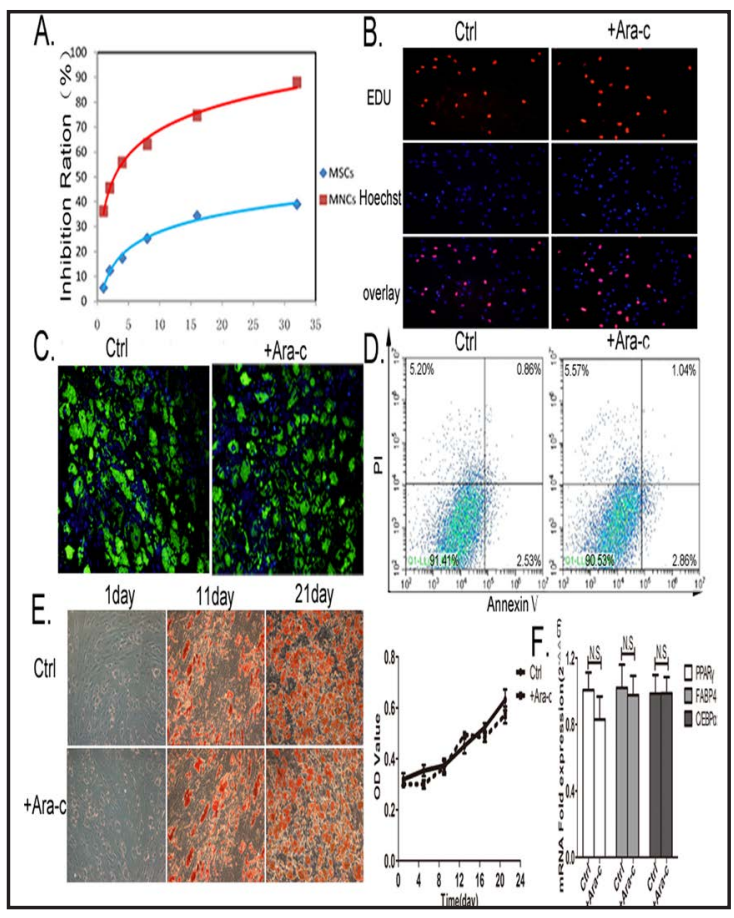

the supernatants of MNCs treated with Ara-C were collected and added to the adipose induction culture (conditioned medium, CM). The number of lipid droplets was significantly reduced compared with the control group, and the rate of adipogenesis decreased after the supernatant from MNCs treated with Ara-C was added (Fig. 3B). This result was confirmed in a PCR assay of adipogenesis-related genes (Fig. 3C). Collectively, our observations clearly suggested that MNCs may secrete some types of soluble cytokines during chemotherapy that play an important role in inhibiting adipogenesis.

$$
\begin{array}{lcr}
\text { GDF15 } & \text { is } & \text { secreted } \\
\text { by } & \text { MNCs } & \text { after }
\end{array}
$$

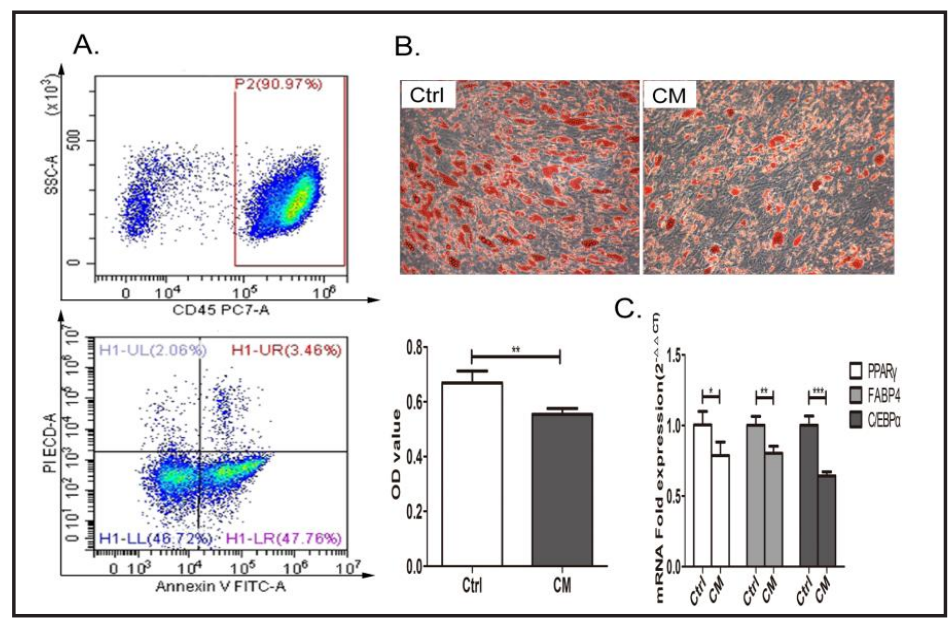

Fig. 3.The supernatant from MNCs exposed to chemotherapy affect adipogenesis. (A) Flow cytometry analysis of the apoptosis of CD45+ cells isolated from the BM of controls.(B) The adipocyte content in the controls (Ctrl) and groups exposed to supernatant from MNCs (condition mediam, CM ) treated with Ara-C $(2 \mu \mathrm{M})$ in adipose induction medium were detected by OD value after ORO staining. Images were obtained at 200× magnification. (C) qPCR analysis of the expression of adipocyte-specific genes (PPAR $\gamma$, FABP4 and C/EBP $\alpha$ ) in adipocytes from the controls and groups exposed to the supernatant from MNCs treated with or without Ara-C $(2 \mu \mathrm{M}) .{ }^{*} \mathrm{p}<0.05,{ }^{* *} \mathrm{p}<0.01,{ }^{* * *} \mathrm{p}<0.001$.

chemotherapy and contributes to the inhibitory effect on adipogenic differentiation

The supernanant of MNCs that received chemotherapy affected adipogenic differentiation. Differentially expressed genes in MNCs treated with or without Ara-C were initially examined using microarray-based profiling to identify cytokines that may participate 


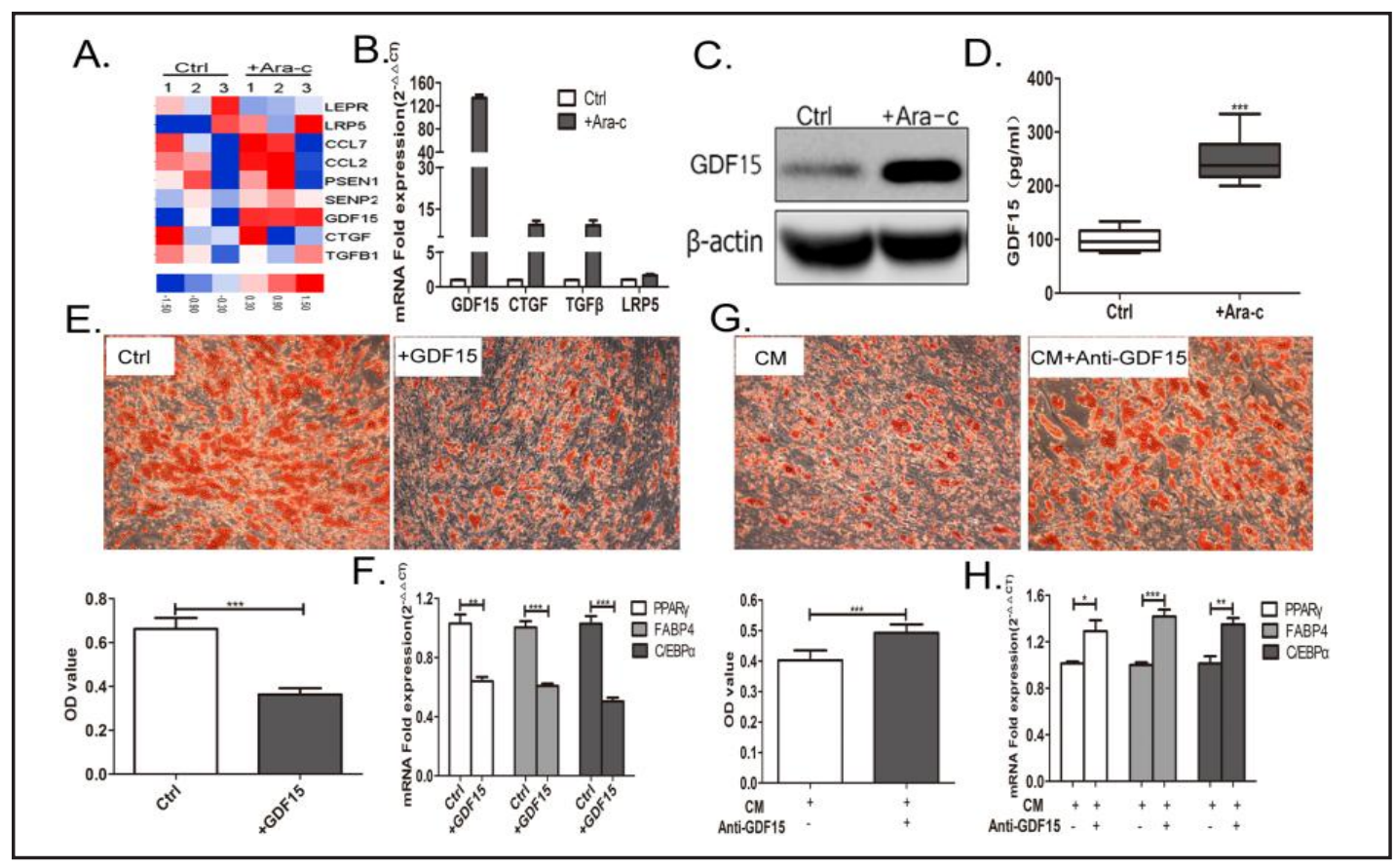

Fig. 4. GDF15 secreted by MNCs in respond to chemotherapy contributes to the inhibitory effect on adipogenic differentiation. (A) Microassay of MNCs treated with or without Ara-C ( $2 \mu \mathrm{M}, \mathrm{n}=3)$. (B) qPCR validation of cytokines expression in MNCs treated with or without Ara-C $(2 \mu \mathrm{M})$. (C)Western blotting validation of GDF15 upregulation in MNCs treated with or without Ara-C $(2 \mu \mathrm{M})$. (D) ELISA validation of GDF15 upregulation in the supernatants of MNCs treated with or without Ara-C $(2 \mu \mathrm{M})$. (E) The adipocyte content in cells exposed to adipose induction medium treated with or without rhGDF15 $(100 \mathrm{ng} / \mathrm{mL})$ was detected by determining the OD value after ORO staining. Images were obtained at $200 \times$ magnification. (F) qPCR analysis of the expression of adipocyte-specific genes in adipocytes exposed to adipose induction medium treated with or without rhGDF15. (G) The adipocyte content from Condition Mediam (CM), added with or without anti-GDF15 antibody ( $4 \mu \mathrm{g} / \mathrm{mL}$ ) detected by the OD value after Oil Red O (ORO) staining. Images obtained at a 200×magnification. (H) qPCR analysis of adipo-specific genes in adipocytes from adipose induction medium treated with or without anti-GDF15.

in inhibiting adipogenic differentiation. Gene expression levels of some cytokines were markedly upregulated, particularly GDF15, which was remarkably upregulated ( $>5.49$-fold) (Fig. 4A), indicating that MNCs might secrete these factors into the BM microenvironment in response to therapy. The array data were also validated by real-time PCR for genes associated with adipogenic differentiation (Fig. 4B). Furthermore, GDF15 expression was obviously upregulated in the treated group, according to western blotting data (Fig. 4C). A greater amount of GDF15 was released into the supernatant of MNCs treated with Ara-C (Fig. $4 \mathrm{D}$ ), suggesting that it may exert a vital biological function. Subsequently, we investigated whether GDF15 endowed cells with resistance to adipogenesis. As shown in Fig. 4E-H, adipogenesis was significantly inhibited in cells treated with rhGDF15, whereas the human neutralizing anti-GDF15 antibody partially attenuated the inhibitory effect, as exhibited by ORO staining and PCR results. Based on these results, GDF15 derived from MNCs after chemotherapy contributed to the inhibitory effect on adipogenic differentiation.

GDF15 is associated with the reduced marrow adipocyte volume in patients with AML-CR

Given the inhibitory effect of GDF15 on adipogenic differentiation in vitro, a correlation analysis was performed to assess the relationship between the GDF15 level and the adipocyte volume. As shown in Fig. 5A, GDF15 was overexpression in samples from patients with AMLCR compared with samples from the control group. In addition, a large amount of GDF15 was released into the serum from the BM (Fig. 5B). Immunoassays were performed on serial 
Liu et al.: Chemotherapy Prevents AML Relapse by Indirectly Regulating Adipogenesis

BM sections from the same sample to visualize GDF15 in the BM and determine whether the MNCs from patients with AML-CR secreted GDF15. The results showed that GDF15 exhibited a scattered distribution in nucleated cells in the BM biopsy, indicating that MNCs from patients with AML-CR might secrete GDF15. In contrast, GDF15 was rarely observed in the control samples (Fig. 5C). Furthermore, a higher GDF15 level in BM samples was associated with a reduced adipocyte content in patients with AML during CR, according to the statistical analyses ( $p=0.031$, Fig. 5D). Based on the experimental results, GDF15 secreted from MNCs in response to chemotherapy played an important role in adipogenesis in patients with AML-CR.

\section{Discussion}

In the present study, we investigated the effects of adipocytes on the prognosis of patients with AML during CR by focusing on the changes in marrow adipocyte volume after chemotherapy. The marrow adipocyte volume was reduced in patients with AML-CR compared to controls, and adipocyte content showed an inverse relationship with RFS. The reduced adipocyte volume was not directly derived from MSCs or the lipid differentiation process. Instead, it was due to GDF15, which was secreted by haematopoietic cells in the BM after chemotherapy and inhibited adipogenesis. Thus, the marrow adipocyte volume may be helpful in evaluating the relapse of patients with AML-CR and GDF15-mediated interference in adipogenesis may enable the development of new perspective treatments for AML.

AML blasts induce lipolysis to support the survival and proliferation of primary AML and significantly reduce the number of marrow adipocytes [24, 25]. Currently, few studies have examined the effects of marrow adipocytes on the prognosis of patients with AML. By quantitatively analysing the adipocyte volume, we are the first to show that the marrow adipocyte content was reduced in samples from patients in remission compared with samples from the control group. Meanwhile, a significantly lower marrow adipocyte volume persisted in patients experiencing continuous remission than in the recurrence group (Fig. 1A and 1F). According to the result of statistical analysis, these changes were not correlated with BM precursors, suggesting that the reduced adipocyte volume in patients with AML-CR might be an independent process. Subsequently, Cox regression analyses revealed that the marrow adipocyte volume was a risk factor for relapse in patients with AML-CR. An increased adipocyte content was associated with a shorter

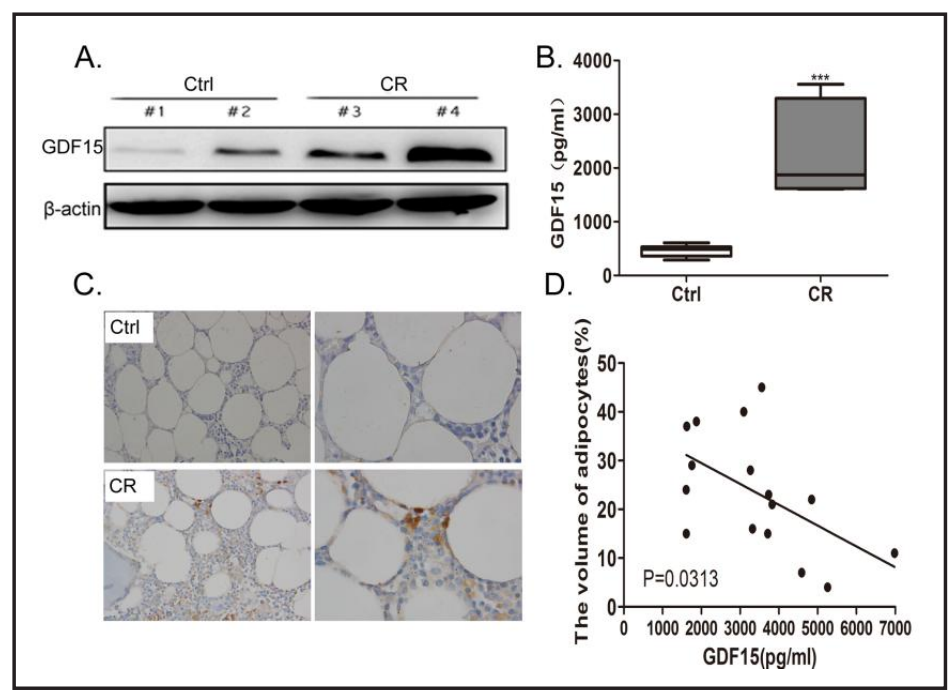

Fig. 5. GDF15 is expressed at high levels and is associated with the adipocyte volume in patients with AML-CR. (A)Western blot analysis of the levels of GDF15 protein levels in MNCs from controls (Ctrl) and patients with AML-CR (CR). (B) ELISA measurement of GDF15 expression in MNCs from controls and patients with AML-CR. (C) Immunocytochemistry assay evaluation of GDF15 expression in sections from patients with AML-CR. Images were obtained at 400× and 1000× magnifications. (D)Analysis of correlation between the adipocyte volume and the GDF15 expression level in the BM of patients with AML during CR ( $n=16, p=0.03)$. 
Liu et al.: Chemotherapy Prevents AML Relapse by Indirectly Regulating Adipogenesis

RFS and an increased risk of relapse. These results are consistent with previous studies showing that adipocytes provide more energy to promote the proliferation of tumor cells. Furthermore, adipocytes play a role in chemotherapy resistance by impairing the antitumor effects of chemotherapeutics and contributing to leukaemia cell survival [26]. Moreover, CD36+ leukaemic stem cells (LSCs) are strikingly enriched in gonadal adipose tissue and are protected from chemotherapy by the adipose tissue niche [27]. Thus, we postulate that a chemo-protective niche in a pool of AML cells might cause relapse, which may require further animal experiments for verification.

The possible reasons for the decreased volume of marrow adipocytes were further explored. Leukaemic cells were recently shown to secrete a large amount of chemotactic factors and growth factors, particularly GDF15, in patients with AML who were receiving induction therapy [10]. This finding prompted us to evaluate the effect of chemotherapy on haematopoietic cells in patients with AML, since leukaemia cells almost completely disappear during remission stage. Indeed, our results showed that the GDF15 level acutely increased in the cellular supernatant of MNCs after cytarabine treatment, and the contribution of MNCs to high GDF15 expression was strongly supported by the gene expression profiles. Meanwhile, GDF15 expression was detected in the BM biopsies from patients with AML-CR. These findings confirmed our hypothesis that GDF15 was mainly secreted by BM MNCs after chemotherapy, which may be an inflammatory or stress response caused by chemotherapy.

Additionally, according to the correlation analysis, the GDF15 expression level was closely related to the marrow adipocyte volume in patients with AML-CR (Fig. 5D). Recently, druginduced GDF15 expression has been shown to markedly inhibit the adipocyte differentiation of mouse 3T3-L1 cells $[20,28]$. Similar results were obtained in our study. Adipogenesis was significantly blocked by the addition of GDF15, whereas depletion of GDF15 using anti-GDF15 antibody partially rescued adipogenic differentiation. Taken together, GDF15 secreted by MNCs in response to chemotherapy contributes to the inhibitory effect on adipogenic differentiation. Furthermore, the WNT signaling pathway exerts an inhibitory effect on the induction of MSC adipogenic differentiation [29, 30]. Indeed, the expression of the co-receptor of the classic WNT signalling pathway, low-density lipoprotein receptorrelated protein 5 (LRP5), was significantly upregulated in the present study (Fig. 4A and 4B), indicating that the WNT/LRP5 signalling pathway may participate in the adipogenic process. However, further investigations are needed to confirm this result.

Normal hematopoietic cells died during the administration of chemotherapy. Meanwhile, inflammatory factors were secreted by these cells, which regulated adipogenic differentiation and indirectly prevented relapse. Thus, consolidation or maintenance therapy is still needed, even in patients who have acquired molecular level of remission [31].However, we have not clearly determined whether the marrow adipocyte volume of patients who have achieved long-term remission without chemotherapy return to normal level and further observations are need to verify this result.

\section{Conclusion}

Taken together, in this retrospective study, our findings demonstrate that a decreased adipocyte volume is associated with longer RFS in patients with AML-CR. Furthermore, GDF15 is secreted by MNCs in response to chemotherapy and partially blocks the adipogenic process of MSCs, which may exert synergistic effects on strengthening the chemotherapeutic efficacy and predicting good outcomes for patients with AML during CR. Thus, the combinations of strategies targeting GDF15 activity with conventional chemotherapy drugs are expected to provide a novel therapeutic approach for AML patients during CR.

\section{Acknowledgements}

This study was funded by grants from the National Natural Science Foundation of China (Grant No. 81570135), National Science and Technology Major Equipment Projects of 


\title{
Cellular Physiology Cell Physiol Biochem 2018;45:2389-2400

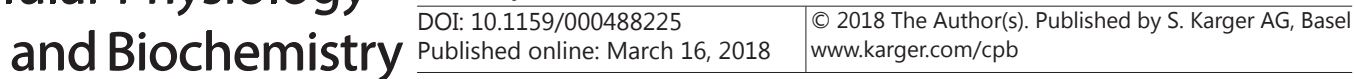

Liu et al.: Chemotherapy Prevents AML Relapse by Indirectly Regulating Adipogenesis

\author{
China (Grant No.2013YQ03065109) and Science and Technology Commission of Shanghai \\ Municipality (Grant No.14142200700).
}

\section{Disclosure Statement}

The authors declare that they have no conflicts of interest.

\section{References}

1 Tavor S, Petit I: Can inhibition of the SDF-1/CXCR4 axis eradicate acute leukemia? Semin Cancer Biol 2010;20:178-185.

$>2$ Sarkozy C, Gardin C, Gachard N, Merabet F, Turlure P, Malfuson JV, Pautas C, Micol JB, Thomas X, Quesnel B, Celli-Lebras K, Preudhomme C, Terre C, Fenaux P, Chevret S, Castaigne S, Dombret H: Outcome of older patients with acute myeloid leukemia in first relapse. Am J Hematol 2013;88:758-764.

-3 Chiarini F, Lonetti A, Evangelisti C, Buontempo F, Orsini E, Evangelisti C, Cappellini A, Neri LM, McCubrey JA, Martelli AM: Advances in understanding the acute lymphoblastic leukemia bone marrow microenvironment: From biology to therapeutic targeting. Biochim Biophys Acta 2016;1863:449-463.

4 Greim H, Kaden DA, Larson RA, Palermo CM, Rice JM, Ross D, Snyder R: The bone marrow niche, stem cells, and leukemia: impact of drugs, chemicals, and the environment. Ann N Y Acad Sci 2014;1310:7-31.

5 Nguyen TV, Melville A, Nath S, Story C, Howell S, Sutton R, Zannettino A, Revesz T: Bone Marrow Recovery by Morphometry during Induction Chemotherapy for Acute Lymphoblastic Leukemia in Children. PLoS One 2015;10:e0126233.

6 Gelelete CB, Pereira SH, Azevedo AM, Thiago LS, Mundim M, Land MG, Costa ES: Overweight as a prognostic factor in children with acute lymphoblastic leukemia. Obesity (Silver Spring) 2011;19:1908-1911.

-7 Steiner RM, Mitchell DG, Rao VM, Schweitzer ME: Magnetic resonance imaging of diffuse bone marrow disease. Radiol Clin North Am 1993;31:383-409.

-8 Cheson BD, Bennett JM, Kopecky KJ, Buchner T, Willman CL, Estey EH, Schiffer CA, Doehner H, Tallman MS, Lister TA, Lo-Coco F, Willemze R, Biondi A, Hiddemann W, Larson RA, Lowenberg B, Sanz MA, Head DR, Ohno R, Bloomfield CD: Revised recommendations of the International Working Group for Diagnosis, Standardization of Response Criteria, Treatment Outcomes, and Reporting Standards for Therapeutic Trials in Acute Myeloid Leukemia. J Clin Oncol 2003;21:4642-4649.

-9 Golay J, Cuppini L, Leoni F, Mico C, Barbui V, Domenghini M, Lombardi L, Neri A, Barbui AM, Salvi A, Pozzi P, Porro G, Pagani P, Fossati G, Mascagni P, Introna M, Rambaldi A: The histone deacetylase inhibitor ITF2357 has anti-leukemic activity in vitro and in vivo and inhibits IL- 6 and VEGF production by stromal cells. Leukemia 2007;21:1892-1900.

10 Duan CW, Shi J, Chen J, Wang B, Yu YH, Qin X, Zhou XC, Cai YJ, Li ZQ Zhang F, Yin MZ, Tao Y, Mi JQ Li LH, Enver T, Chen GQ Hong DL: Leukemia propagating cells rebuild an evolving niche in response to therapy. Cancer Cell 2014;25:778-793.

11 Rosen CJ, Ackert-Bicknell C, Rodriguez JP, Pino AM: Marrow fat and the bone microenvironment: developmental, functional, and pathological implications. Crit Rev Eukaryot Gene Expr 2009;19:109-124.

12 Caers J, Deleu S, Belaid Z, De Raeve H, Van Valckenborgh E, De Bruyne E, Defresne MP, Van Riet I, Van Camp B, Vanderkerken K: Neighboring adipocytes participate in the bone marrow microenvironment of multiple myeloma cells. Leukemia 2007;21:1580-1584.

13 Castro-Munozledo F, Beltran-Langarica A, Kuri-Harcuch W: Commitment of 3T3-F442A cells to adipocyte differentiation takes place during the first 24-36 $\mathrm{h}$ after adipogenic stimulation: TNF-alpha inhibits commitment. Exp Cell Res 2003;284:163-172.

14 Wallin U, Glimelius B, Jirstrom K, Darmanis S, Nong RY, Ponten F, Johansson C, Pahlman L, Birgisson H: Growth differentiation factor 15: a prognostic marker for recurrence in colorectal cancer. Br J Cancer 2011;104:1619-1627.

15 Koopmann J, Rosenzweig CN, Zhang Z, Canto MI, Brown DA, Hunter M, Yeo C, Chan DW, Breit SN, Goggins M: Serum markers in patients with resectable pancreatic adenocarcinoma: macrophage inhibitory cytokine 1 versus CA19-9. Clin Cancer Res 2006;12:442-446. 


\section{Cellular Physiology Cell Physiol Biochem 2018;45:2389-2400

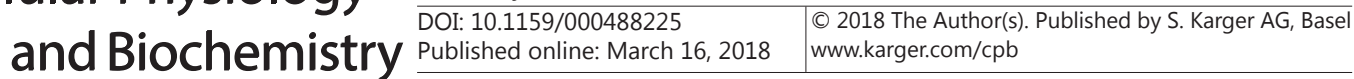

Liu et al.: Chemotherapy Prevents AML Relapse by Indirectly Regulating Adipogenesis

-16 Staff AC, Trovik J, Eriksson AG, Wik E, Wollert KC, Kempf T, Salvesen HB: Elevated plasma growth differentiation factor-15 correlates with lymph node metastases and poor survival in endometrial cancer. Clin Cancer Res 2011;17:4825-4833.

17 Brown DA, Lindmark F, Stattin P, Balter K, Adami HO, Zheng SL, Xu J, Isaacs WB, Gronberg H, Breit SN, Wiklund FE: Macrophage inhibitory cytokine 1: a new prognostic marker in prostate cancer. Clin Cancer Res 2009;15:6658-6664.

-18 Husaini Y, Lockwood GP, Nguyen TV, Tsai VW, Mohammad MG, Russell PJ, Brown DA, Breit SN: Macrophage inhibitory cytokine-1 (MIC-1/GDF15) gene deletion promotes cancer growth in TRAMP prostate cancer prone mice. PLoS One 2015;10:e0115189.

19 Bootcov MR, Bauskin AR, Valenzuela SM, Moore AG, Bansal M, He XY, Zhang HP, Donnellan M, Mahler S, Pryor K, Walsh BJ, Nicholson RC, Fairlie WD, Por SB, Robbins JM, Breit SN: MIC-1, a novel macrophage inhibitory cytokine, is a divergent member of the TGF-beta superfamily. Proc Natl Acad Sci U S A 1997;94:11514-11519.

20 Yanagitai M, Kitagawa T, Okawa K, Koyama H, Satoh T: Phenylenediamine derivatives induce GDF-15/MIC-1 and inhibit adipocyte differentiation of mouse 3T3-L1 cells. Biochem Biophys Res Commun 2012;417:294298.

21 Creutzig U, Kaspers GJ: Revised recommendations of the International Working Group for diagnosis, standardization of response criteria, treatment outcomes, and reporting standards for therapeutic trials in acute myeloid leukemia. J Clin Oncol 2004;22:3432-3433.

22 Meulle A, Salles B, Daviaud D, Valet P, Muller C: Positive regulation of DNA double strand break repair activity during differentiation of long life span cells: the example of adipogenesis. PLoS One 2008;3:e3345.

23 Schmid C, Schleuning M, Schwerdtfeger R, Hertenstein B, Mischak-Weissinger E, Bunjes D, Harsdorf SV, Scheid C, Holtick U, Greinix H, Keil F, Schneider B, Sandherr M, Bug G, Tischer J, Ledderose G, Hallek M, Hiddemann W, Kolb HJ: Long-term survival in refractory acute myeloid leukemia after sequential treatment with chemotherapy and reduced-intensity conditioning for allogeneic stem cell transplantation. Blood 2006;108:1092-1099.

24 Lu W, Weng W, Zhu Q, Zhai Y, Wan Y, Liu H, Yang S, Yu Y, Wei Y, Shi J: Small bone marrow adipocytes predict poor prognosis in acute myeloid leukemia. Haematologica 2018;103:e21-e24.

25 Shafat MS, Oellerich T, Mohr S, Robinson SD, Edwards DR, Marlein CR, Piddock RE, Fenech M, Zaitseva L, Abdul-Aziz A, Turner J, Watkins JA, Lawes M, Bowles KM, Rushworth SA: Leukemic blasts program bone marrow adipocytes to generate a pro-tumoral microenvironment. Blood. 2017;129(10):1320-1332

26 Behan JW, Yun JP, Proektor MP, Ehsanipour EA, Arutyunyan A, Moses AS, Avramis VI, Louie SG, Butturini A, Heisterkamp N, Mittelman SD: Adipocytes impair leukemia treatment in mice. Cancer Res 2009;69:78677874.

27 Ye H, Adane B, Khan N, Sullivan T, Minhajuddin M, Gasparetto M, Stevens B, Pei S, Balys M, Ashton JM, Klemm DJ, Woolthuis CM, Stranahan AW, Park CY, Jordan CT: Leukemic Stem Cells Evade Chemotherapy by Metabolic Adaptation to an Adipose Tissue Niche. Cell Stem Cell 2016;19:23-37.

28 Chrysovergis K, Wang X, Kosak J, Lee SH, Kim JS, Foley JF, Travlos G, Singh S, Baek SJ, Eling TE: NAG-1/GDF15 prevents obesity by increasing thermogenesis, lipolysis and oxidative metabolism. Int J Obes (Lond) 2014;38:1555-1564.

-29 Ross SE, Hemati N, Longo KA, Bennett CN, Lucas PC, Erickson RL, MacDougald OA: Inhibition of adipogenesis by Wnt signaling. Science 2000;289:950-953.

-30 Christodoulides C, Lagathu C, Sethi JK, Vidal-Puig A: Adipogenesis and WNT signalling. Trends Endocrinol Metab 2009;20:16-24.

-31 Battipaglia G, Ruggeri A, Massoud R: Efficacy and feasibility of sorafenib as a maintenance agent after allogeneic hematopoietic stem cell transplantation for Fms-like tyrosine kinase 3-mutated acute myeloid leukemia. 2017;123:2867-2874. 